\title{
Long-lasting extrapyramidal symptoms after multiple injections of paliperidone palmitate to treat schizophrenia
}

This article was published in the following Dove Press journal:

Neuropsychiatric Disease and Treatment

\author{
Ryohei Takada \\ Kazuhiko Yamamuro \\ Toshifumi Kishimoto \\ Department of Psychiatry, Nara \\ Medical University School of \\ Medicine, Kashihara, Japan
}

Correspondence: Kazuhiko Yamamuro Department of Psychiatry, Nara Medical University School of Medicine, 840 Shijo-cho, Kashihara, Nara 634-852I, Japan

$\mathrm{Tel}+8 \mathrm{I} 74422305$ I

Fax +8I 744223854

Email muro@naramed-u.ac.jp
Purpose: Antipsychotic drug treatment can potentially lead to adverse events such as extrapyramidal symptoms (EPSs). If these events persist, they lower patient quality of life. The purpose of this report is to present our experience with such a case, as an example to encourage further study.

Patients and methods: The patient was a 64-year-old male who had been diagnosed with schizophrenia that had been treated with oral antipsychotics for over 40 years. Due to lack of compliance, he was treated with the long-acting injectable antipsychotic, paliperidone palmitate, but developed persistent EPSs after 6 months. He was subsequently treated and monitored in our inpatient facility.

Results: Antipsychotic treatment was stopped. After 6 weeks, the patient's psychosis had worsened, but his EPSs remained unchanged. Levodopa treatment was then started (up to $400 \mathrm{mg} / \mathrm{d}$ ), which improved his EPSs, so he was restarted on oral antipsychotic therapy (aripiprazole, 6-18 mg/d). His psychotic symptoms improved over 2 months. The improvements in both psychosis and EPSs remained stable. Dopamine transporter scans revealed moderate dopamine transporter loss in the striatum, and computed tomography revealed no sign of brain abnormalities, suggesting that the patient was susceptible to dopamine reductions.

Conclusion: Paliperidone palmitate can induce EPSs, even if injected only a few times. Although the characteristics of the drug are potential causes, the patient's predisposition to dopamine perturbations can also influence the outcome. Therefore, adequate awareness is required before injection.

Keywords: schizophrenia, long-lasting injectable, dopamine, levodopa

\section{Introduction}

Schizophrenia, which is a chronic and severe mental disorder characterized by positive symptoms, negative symptoms, and progressive cognitive decline, is relatively common and is one of the top 25 leading causes of disability worldwide. ${ }^{1}$ Schizophrenia greatly influences a patient's quality of life, social skills, and mortality, and creates a huge social and economic burden. ${ }^{2}$ Unfortunately, the prognosis and outcomes worsen with each relapse due to poor treatment adherence. ${ }^{3,4}$ In fact, a previous study revealed that approximately $50 \%$ of patients with schizophrenia do not fully comply with treatment. ${ }^{5}$ To address this matter, the appropriate use of long-acting injectable (LAI) antipsychotics, especially atypical antipsychotics, has the potential to increase treatment adherence because there is no need to take daily oral antipsychotics. ${ }^{6}$ Moreover, the higher and consistent bioavailability of LAI antipsychotics can reduce fluctuating serum drug levels and improve peak serum levels, resulting in the reduction in several adverse side 
effects such as extrapyramidal symptoms (EPSs). ${ }^{7}$ However, LAI antipsychotics also have serious disadvantages because, side effects tend to persist once they appear, rendering their management very difficult. ${ }^{8}$ Paliperidone palmitate (PP), the LAI form of paliperidone, is widely used and approved due to its efficacy and safety. ${ }^{9}$

To the best of our knowledge, only one previous case study reported that PP induced EPSs for 5 months after a single injection. ${ }^{10}$ No studies to date, however, have reported the background of patients with schizophrenia in the case of PP-induced EPSs after 6 months. Consequently, this report also addresses the causes of EPSs by using several imaging techniques, namely magnetic resonance imaging (MRI) and dopamine transporter (DaT) scans, in the case of PP-induced EPSs in a patient with schizophrenia.

\section{Case presentation}

Patient A was a 64-year-old man who began experiencing delusions of persecution and auditory hallucinations at the age of 22 years. When he was 23 years old, he visited a local clinic and was diagnosed with schizophrenia. He was prescribed several antipsychotics, including haloperidol, risperidone (6 mg/d), olanzapine $(20 \mathrm{mg} / \mathrm{d})$, and aripiprazole (24 mg/d); the drugs were administered nonconcurrently. Although he was continuously prescribed each antipsychotic, he did not consistently adhere to treatment due to delusions of being poisoned. However, he had no complaint about EPSs at this point. He was repeatedly hospitalized and discharged from a local psychiatric hospital because his symptoms had exhibited cyclical remissions and relapses. When he was 64 years old, due to poor adherence and relapse of psychotic symptoms, he was started on PP injections at a dose of $150 \mathrm{mg}$. Then, he received a second $100 \mathrm{mg}$ dose 1 week later, followed by $100 \mathrm{mg}$ monthly injections.

After the initiation of injection, his psychotic symptoms, such as delusion of persecution and auditory hallucinations, gradually improved. However, after 6 months of consecutive PP injections, severe EPSs appeared and he began to experience difficulty walking and speaking fluently. Because these severe EPSs did not improve upon cessation of PP injections, he was admitted to our psychiatric hospital. His psychological symptoms were assessed using the Japanese version of the Positive And Negative Syndrome Scale (PANSS). Higher PANSS scores indicate more severe psychotic symptoms. We also used the Drug-Induced Extrapyramidal Symptoms Scale (DIEPSS), which was developed to measure druginduced movement disorders. ${ }^{11,12}$ We used the total scores on the DIEPSS for analysis. The interrater reliability, test-retest reliability, and concurrent validity with other EPS rating scales have been excellent. ${ }^{11,12}$

There were neither previous reports of premorbid symptoms, nor a family history of psychosis or other mental disorders. Computed tomography revealed no signs of brain abnormalities. He exhibited hand tremors, lead-pipe rigidity, gait instability, sialorrhea, and oral dyskinesia, in addition to his severe hallucinatory delusions (PANSS score of 125, DIEPSS score of 22). Despite receiving no antipsychotics over the 6 weeks after admission, we observed no improvement in his EPSs (DIEPSS score of 21) and no improvement in his psychotic symptoms (PANSS score of 138). Therefore, we started levodopa at a dose that increased to $400 \mathrm{mg} / \mathrm{d}$. After 1 month, the EPSs gradually improved (DIEPSS score of 13), so aripiprazole was started at $6 \mathrm{mg} / \mathrm{d}$, and then increased to $18 \mathrm{mg} / \mathrm{d}$, while actively monitoring EPSs for signs of deterioration. His psychotic symptoms gradually improved and EPSs did not worsen after 2 months (PANSS score of 88, DIEPSS score of 5). Finally, neither his EPSs nor symptoms had deteriorated over the following 6 months. His prognosis is demonstrated in Figure 1. Importantly, he had no the other side effects including liver/renal dysfunction.

Interestingly, he showed moderate DaT loss in the striatum, as assessed by DaT scan, and atrophy of the dorsal brain stem, as assessed by MRI. These findings suggest that he was at least vulnerable to dopamine reductions, although he might not fulfill the diagnostic criteria of Parkinson's disease.

\section{Discussion}

To the best of our knowledge, only a single case study has reported that PP induced 5-month persistent EPSs after a single injection. ${ }^{10}$ Oral paliperidone extended-release is generally an effective and well-tolerated therapy ${ }^{13}$ and one of the first-line drugs to treat patients with schizophrenia. The incidence rates of EPSs, except for hyperkinesia, were found to be lower with PP than with oral paliperidone. ${ }^{14}$ However, the interesting finding in our case report was that PP induced 6-month persistent EPSs after several PP injections.

Previous positron emission tomography data combined with mean plasma concentration determinations have indicated that optimal clinical response occurs when $65 \%-80 \%$ of striatal dopamine-2 (D2) receptors are occupied. ${ }^{15,16}$ However, the risk of EPSs increases notably at D2 occupancy levels above $80 \% .^{15,16}$ In a previous report, the dose-adjusted median serum concentrations of paliperidone varied considerably for each patient. ${ }^{17}$ In addition, the serum concentration may continue to increase long after 6 months. ${ }^{17}$ Therefore, in our case, it took approximately 6 months to reach a steady 


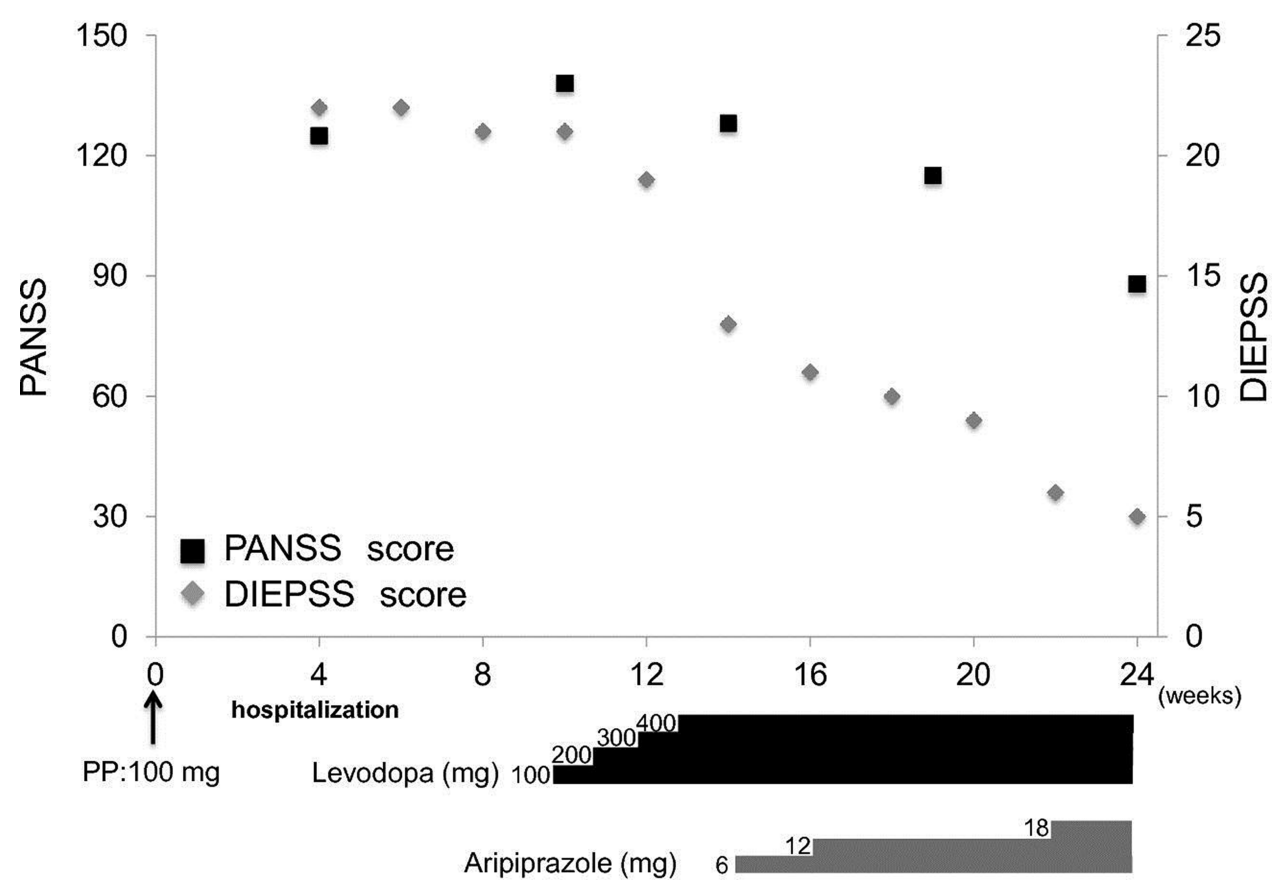

Figure I Course of the PANSS and DIEPSS.

Abbreviations: DIEPSS, Drug-Induced Extrapyramidal Symptoms Scale; PANSS, Positive And Negative Syndrome Scale; PP, paliperidone palmitate.

state, and severe EPSs might appear as the occupation rate of the D2 receptor in the striatum exceeded $80 \%$.

Moreover, in our case, EPSs persisted for more than 6 months since the last injection, although the serum concentration of paliperidone was expected to gradually subside. PP is an insoluble ester of paliperidone and dissolves very slowly in the interstitial fluids at the site of injection; paliperidone enters the systemic circulation over an extended period of time (125-245 days). ${ }^{18}$ In addition, the serum half-life is relatively long, roughly $25-49$ days. ${ }^{9}$ In the case of such extended-release medications, it is necessary to consider that they can persist in the body for approximately 4-5 times longer than their half-life, even after discontinuation. Therefore, considering previously reported data and our case report collectively, paliperidone could remain in the body after cessation of treatment, and this could cause persistent EPSs.

In this case, a DaT scan revealed moderate DaT loss in the striatum, in addition to which MRI revealed atrophy of the dorsal brain stem. This suggested that the patient was vulnerable to EPS development with antipsychotic drug use. When we examined his medical history in detail at a later time, we found, for the first time, that he had periodically complained of spontaneous EPSs 4 years ago. Importantly, although he has been prescribed many types of antipsychotic, he has not experienced EPSs. Therefore, age might be one of the obvious risk factors for EPSs, ${ }^{19}$ since dopamine concentrations decrease and nigral cells degenerate with age.$^{20}$ These findings suggest that his predisposed vulnerability to antipsychotic drug use might be one of the causes of the persistent EPSs.

There are several methods for management of antipsychotic-induced EPSs, including stopping the antipsychotics in use, switching to other antipsychotics, and initiation of antiparkinson medication. Among antiparkinson medications, levodopa is an effective drug for management of antipsychotic-induced EPSs. ${ }^{21}$ In our case, EPSs did not improve until after levodopa treatment was initiated. This supports the efficacy of levodopa against antipsychoticinduced EPSs.

As the present report involves only one case, the major limitation of our study is the small sample size. Also, we have not been able to confirm the patient's medication adherence. Future studies should continue to assess the use of PP in patients with schizophrenia in controlled samples including younger participants.

\section{Conclusion}

This case suggests that PP can cause EPSs in patients with schizophrenia, even if they are injected with PP only a few times. Further studies with larger sample sizes are needed to clarify this causal relationship. In order to avoid the occurrence of unexpected EPSs, it is necessary to sufficiently investigate the medical history and the physical and neurological symptoms of individual patients before administration 
of LAI antipsychotics. The evaluation of the vulnerability to antipsychotic drugs is especially important. We should also be aware of the possibility that unexpected EPSs may occur after several injections of LAI antipsychotics.

\section{Ethical consideration}

As we encountered this case during regular clinical practice, the need for ethics approval and consent was waived. We obtained written informed consent for the publication of this case report from the patient.

\section{Data sharing statement}

Data sharing was not applicable to this article as no datasets were generated or analyzed during the current study.

\section{Acknowledgments}

We would like to thank the patient and his family for their cooperation. This case report did not receive any specific grant from funding agencies in the public, commercial, or not-for-profit sectors.

\section{Author contributions}

RT collected data and wrote the first draft of the manuscript. KY and TK supervised the project, were critically involved in its design, and assisted in editing the final manuscript. All authors contributed toward data analysis, drafting, and revising the paper, and all agreed to be accountable for all aspects of the work.

\section{Disclosure}

The authors report no conflicts of interest in this work.

\section{References}

1. Global Burden of Disease Study 2013 Collaborators. Global, regional, and national incidence, prevalence, and years lived with disability for 301 acute and chronic diseases and injuries in 188 countries, 1990-2013: a systematic analysis for the Global Burden of Disease Study 2013. Lancet. 2015;386(9995):743-800.

2. Chong HY, Teoh SL, Wu DB, Kotirum S, Chiou CF, Chaiyakunapruk N. Global economic burden of schizophrenia: a systematic review. Neuropsychiatr Dis Treat. 2016;12:357-373.

3. Kane JM. Treatment strategies to prevent relapse and encourage remissionTreatment strategies to prevent relapse and encourage remission. J Clin Psychiatry. 2007;68(Suppl)14:27-30.
4. Lindenmayer JP, Liu-Seifert H, Kulkarni PM, et al. Medication nonadherence and treatment outcome in patients with schizophrenia or schizoaffective disorder with suboptimal prior response. J Clin Psychiatry. 2009;70(7):990-996.

5. Perkins DO. Predictors of noncompliance in patients with schizophrenia. J Clin Psychiatry. 2002;63(12):1121-1128.

6. Kane JM. Strategies for improving compliance in treatment of schizophrenia by using a long-acting formulation of an antipsychotic: clinical studies. J Clin Psychiatry. 2003;64(Suppl):16:34-40.

7. Ereshefsky L, Mascarenas CA. Comparison of the effects of different routes of antipsychotic administration on pharmacokinetics and pharmacodynamics. J Clin Psychiatry. 2003;64(Suppl):16:18-23.

8. Brissos S, Veguilla MR, Taylor D, Balanzá-Martinez V. The role of long-acting injectable antipsychotics in schizophrenia: a critical appraisal. Ther Adv Psychopharmacol. 2014;4(5):198-219.

9. Kramer M, Litman R, Hough D, et al. Paliperidone palmitate, a potential long-acting treatment for patients with schizophrenia. Results of a randomized, double-blind, placebo-controlled efficacy and safety study. Int J Neuropsychopharmacol. 2010;13(5):635-647.

10. Jang S, Woo J. Five Month-Persistent Extrapyramidal Symptoms following a Single Injection of Paliperidone Palmitate: A Case Report. Clin Psychopharmacol Neurosci. 2017;15(3):288-291.

11. Inada $\mathrm{T}$, Yagi G, Miura S. Extrapyramidal symptom profiles in Japanese patients with schizophrenia treated with olanzapine or haloperidol. Schizophr Res. 2002;57(2-3):227-238.

12. Kim JH, Lee BC, Park HJ, Ahn YM, Kang UG, Kim YS. Subjective emotional experience and cognitive impairment in drug-induced akathisia. Compr Psychiatry. 2002;43(6):456-462.

13. Helldin L, Peuskens J, Vauth R, et al. Treatment response, safety, and tolerability of paliperidone extended release treatment in patients recently diagnosed with schizophrenia. Ther Adv Psychopharmacol. 2015; 5(4):194-207.

14. Gopal S, Liu Y, Alphs L, Savitz A, Nuamah I, Hough D. Incidence and time course of extrapyramidal symptoms with oral and long-acting injectable paliperidone: a posthoc pooled analysis of seven randomized controlled studies. Neuropsychiatr Dis Treat. 2013;9:1381-1392.

15. Remington G, Mamo D, Labelle A, et al. A PET study evaluating dopamine D2 receptor occupancy for long-acting injectable risperidone. Am J Psychiatry. 2006;163(3):396-401.

16. Horacek J, Bubenikova-Valesova V, Kopecek M, et al. Mechanism of action of atypical antipsychotic drugs and the neurobiology of schizophrenia. CNS Drugs. 2006;20(5):389-409.

17. Helland A, Spigset O. Serum Concentrations of Paliperidone After Administration of the Long-Acting Injectable Formulation. Ther Drug Monit. 2017;39(6):659-662.

18. Samtani MN, Gopal S, Gassmann-Mayer C, Alphs L, Palumbo JM. Dosing and switching strategies for paliperidone palmitate: based on population pharmacokinetic modelling and clinical trial data. CNS Drugs. 2011;25(10):829-845.

19. Susatia F, Fernandez HH. Drug-induced parkinsonism. Curr Treat Options Neurol. 2009;11(3):162-169.

20. Volkow ND, Ding YS, Fowler JS, et al. Dopamine transporters decrease with age. J Nucl Med. 1996;37(4):554-559

21. Tinazzi M, Antonini A, Bovi T, et al. Clinical and [123I]FP-CIT SPET imaging follow-up in patients with drug-induced parkinsonism. J Neurol. 2009;256(6):910-915.
Neuropsychiatric Disease and Treatment

\section{Publish your work in this journal}

Neuropsychiatric Disease and Treatment is an international, peerreviewed journal of clinical therapeutics and pharmacology focusing on concise rapid reporting of clinical or pre-clinical studies on a range of neuropsychiatric and neurological disorders. This journal is indexed on PubMed Central, the 'PsycINFO' database and CAS,

\section{Dovepress}

and is the official journal of The International Neuropsychiatric Association (INA). The manuscript management system is completely online and includes a very quick and fair peer-review system, which is all easy to use. Visit http://www.dovepress.com/testimonials.php to read real quotes from published authors. 